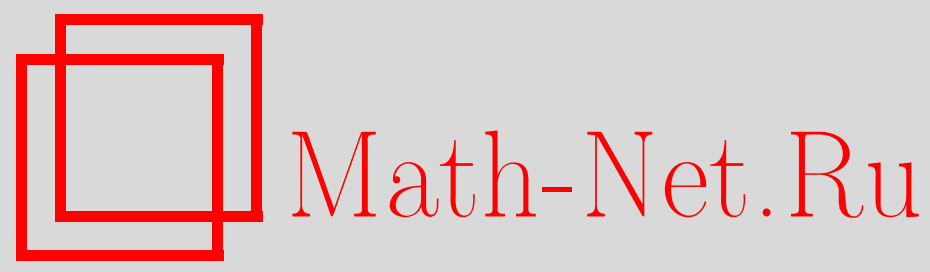

Р. Ш. Оманадзе, Мажорные множества, классы простых множеств и $Q$-полные множества, Матем. заметки, 2002, том 71, выпуск 1, 100-108

DOI: https://doi.org/10.4213/mzm331

Использование Общероссийского математического портала Math-Net.Ru подразумевает, что вы прочитали и согласны с пользовательским соглашением http://www . mathnet.ru/rus/agreement

Параметры загрузки:

IP : 52.205 .19 .152

26 апреля 2023 г., 15:36:22

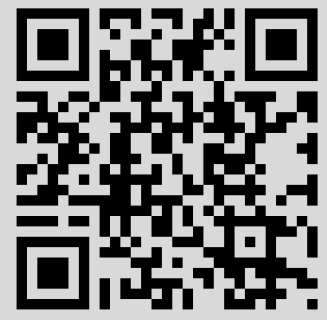




\title{
МАЖОРНЫЕ МНОЖЕСТВА, КЛАССЫ ПРОСТЫХ МНОЖЕСТВ И $Q$-ПОЛНЫЕ МНОЖЕСТВА
}

\section{Р.Ш. Оманадзе}

\begin{abstract}
Доказано, что каждое нерекурсивное рекурсивно перечислимое множество имеет $Q$-полное мажорное подмножество. Определены классы простых множеств, в которых существуют $Q$-полные множества.

Библиография: 16 названий.
\end{abstract}

В работе [1] Херрманом исследованы соотношения по включению между классами простых множеств, и на основе известных результатов разных авторов и самого Херрмана доказана классификационная теорема. Эта теорема вместе с теоремой 2 из [2] дает окончательную картину взаимного расположения некоторьх классов простых множеств.

Из хорошо известной теоремы Деккера (см., например, [3, теорема XVI, с. 182]) и из леммы 1 из [4] следует, что существует $Q$-полное гиперпростое множество. В работах [5] и [6] доказано, что $Q$-полное множество может не быть гиперпростым. Поэтому естественно возникает вопрос: в каких классах простых множеств содержатся $Q$-полные множества? На этот вопрос в данной работе дается ответ для классов простых множеств, представленных в указанной вьше классификационной теореме.

Все употребляемые без определения обозначения и понятия можно найти в [3].

Рекурсивно перечислимое (РП) подмножество $B$ РП множества $A$ называется $\boldsymbol{м} a$ жорныц подмножеством $A\left(B \subset_{m} A\right)$, если $A \backslash B$ - бесконечное множество и для любого РП множества $W$

$$
\bar{A} \subseteq^{*} W \Longrightarrow \bar{B} \subseteq^{*} W .
$$

(Для множеств $X, Y$ мы пишем $X \subseteq^{*} Y$, если $X \backslash Y$ конечно.)

Лахлан [7] доказал, что каждое нерекурсивное РП множество имеет мажорное подмножество. С использование теоремы Лахлана и теоремы 2 из [2] доказывается следующее утверждение.

Теорема 1. Каждое нерекурсивное РП множество $A$ имеет мажорное подмножество $B$ такое, что всякое надмножество $C$ множества $B$, удовлетворяющее условию

$$
C \subset_{m} A,
$$


является $Q-$ полным.

Напомним, что множество $A Q$-сводится к множеству $B(A \leqslant Q \quad B)$, если существует общерекурсивная функция (ОР $\Phi) f$ такая, что

$$
(\forall x)\left(x \in A \Longleftrightarrow W_{f(x)} \subseteq B\right) .
$$

РП множество $A$ называется $Q$-nолным, если каждое РП множество $Q$-сводится к множеству $A$.

РП множество $A$ с бесконечным дополнением назьвается конечно строго гиперпростым м, если не существует ОР $\Phi f$ такой, что

1) $(\forall x)(\forall y)\left(x \neq y \Longrightarrow W_{f(x)} \cap W_{f(y)}=\varnothing\right)$;

2) $(\forall x)\left(W_{f(x)} \cap \bar{A} \neq \varnothing\right)$;

3) $(\forall x)\left(\left|W_{f(x)}\right|<\infty\right)$;

4) $\bigcup_{x \in \omega} W_{f(x)}=\omega$, где $\omega=\{0,1,2, \ldots\}$.

Для доказательства теоремы 1 нам понадобится следующее утверждение.

Лемма 1. Пусть $A, B$ - нерекурсивные РП множсества, $A \subset_{m} B, f$ - одно-однозначная $\mathrm{OP} \Phi, \mathrm{Val} f=B u A_{1}=f^{-1}(A)$. Тогда $A_{1}$ не является конечно строго гиперпростым мнохеством.

ДокАЗАТЕЛЬСтво. Допустим, что выполняются условия леммы. Согласно теореме 3 Лахлана [7] (см. также [3, теорема XX, с. 315]) $A \cup \bar{B}$ не является РП множеством. Тогда существует (см. [8, теорема 1, с. 200]) ОР $\Phi$ такая, что

1) $(\forall x)(\forall y)\left(x \neq y \Longrightarrow W_{g(x)} \cap W_{g(y)}=\varnothing\right)$;

2) $(\forall x)\left(\left|W_{g(x)}\right|<\infty\right)$;

3) $(\forall x)\left(W_{g(x)} \cap(B \backslash A) \neq \varnothing\right)$;

4) $A \cup\left(\bigcup_{x \in \omega} W_{g(x)}\right)=B$.

Пусть $f_{1}$ - одно-однозначная ОР $\Phi, \operatorname{Val} f_{1}=A$ и $\alpha-\mathrm{OP} \Phi$ такая, что

$$
(\forall x)\left(W_{\alpha(x)}=W_{g(x)} \cup\left\{f_{1}(x)\right\}\right) .
$$

Тогда существует (см. [3, с. 105] или [6]) ОРФ $\beta$ такая, что

$$
\begin{gathered}
(\forall x)(\forall y)\left(x \neq y \Longrightarrow W_{\beta(x)} \cap W_{\beta(y)}=\varnothing\right), \\
(\forall x)\left(W_{\beta(x)} \subseteq W_{\alpha(x)}\right), \\
\bigcup_{x \in \omega} W_{\beta(x)}=\bigcup_{x \in \omega} W_{\alpha(x)} .
\end{gathered}
$$

Очевидно, что ОР $\Phi \beta$ удовлетворяет условиям 1)-3) и

$$
B=\bigcup_{x \in \omega} W_{\beta(x)}
$$

Пусть $\gamma-$ ОРФ такая, что

$$
(\forall x)\left(W_{\gamma(x)}=f^{-1}\left(W_{\beta(x)}\right)\right) .
$$

Легко проверить, что ОР $\Phi$ удовлетворяет условиям 1)-4) из определения конечно строго гиперпростого множества. Следовательно, $A_{1}$ не является конечно строго гиперпростьм множеством. Лемма доказана. 
ДоказАТЕЛЬСтво теоремЫ 1. Пусть $A$ - нерекурсивное РП множество, $E \subset_{m} A$, $f$ - одно-однозначная ОР $\Phi, \operatorname{Val} f=A$ и $E_{1}=f^{-1}(E)$. Согласно лемме $1 E_{1}$ не может быть конечно строго гиперпростым множеством. Тогда в силу теоремы 2 из [2] существует РП множество $F, E_{1} \subseteq F$, такое, что всякое РП надмножество множества $F$ с бесконечным дополнением является $Q$-полным. Поэтому для завершения доказательства теоремы 1 достаточно в качестве $B$ взять множество $f(F)$. Теорема доказана.

ЗАмЕчанИЕ. Известно (см. [6] и [9]), что класс $Q$-полных РП множеств совпадает с классом эффективно ускоряемых множеств.

СлЕДСТВИЕ 1. Для каждого нерекурсивного РП множества $M$ существуют $\mathrm{PП} \mathrm{подмножества} A, B, A_{1}$ и $B_{1}$ такие, что

1) $A \subset_{m} B \subset_{m} M u A$ не является $Q$-полным, а $B$ является $Q$-полным;

2) $A_{1} \subset_{m} B_{1} \subset_{m} M u A_{1}, B_{1}-Q$-полные мнохества.

ДокАЗАтЕЛьСтво. 1) Пусть $M$ - нерекурсивное РП множество и $A$ - мажорное подмножество множества $M$, которое не является $Q$-полньм. Такое множество сушествует по теореме 1.1 из [10]. Тогда по теореме 3 из [7] $M \backslash A$ не может быть гипергипериммунным множеством. Поэтому существует ОР $\Phi f$ такая, что

$$
\begin{gathered}
(\forall x)\left(W_{f(x)} \cap(M \backslash A) \neq \varnothing\right), \\
(\forall x)(\forall y)\left(x \neq y \Longrightarrow W_{f(x)} \cap W_{f(y)}=\varnothing\right) .
\end{gathered}
$$

Без ограничения общности можно считать, что $(\forall x)\left(W_{f(x)} \subseteq M\right)$, иначе вместо $f$ взяли бы $g$ такую, что $(\forall x)\left(W_{g(x)}=M \cap W_{f(x)}\right)$.

Для завершения доказательства пункта 1) достаточно рассмотреть в качестве $B$ множество

$$
A \cup\left(\bigcup_{x \in K} W_{f(x)}\right),
$$

где $K$ - креативное множество.

2) Аналогично, для доказательства пункта 2) допустим, что $A_{1}$ есть $Q$-полное мажорное подмножество множества $M$, а

$$
B_{1}=A_{1} \cup\left(\bigcup_{x \in K} W_{f(x)}\right) .
$$

СЛЕДСТВИЕ 2. Для каждого максимального множества $M$ существуют $r$-максимальные $Q$-полные множества $A, B$ такие, что $A \subset_{m} B \subset_{m} M u A \Varangle_{m} B \&$ $B \nless_{m} A$.

ДоКАЗАТЕЛЬСТво. Известно, что мажорное подмножество максимального множества является $r$-максимальным. Дёгтев [11] показал, что если $A-r$-максимальное множество, $B$ - РП множество, $A \subset B, B \backslash A$ и $\bar{B}$ - бесконечные множества, то $A \Varangle_{m} B \&$ $B \Varangle_{m} A$. Согласно утверждению 2) следствия 1 существуют $Q$-полные подмножества $A, B$ множества $M$ такие, что $A \subset_{m} B \subset_{m} M$. 
ПРЕДЛОЖЕНИЕ. Пусть $A-T$-полное РП множество, $H$ - гипергиперпростое множество, $A \subset_{m} H, f$-одно-однозначная $\mathrm{OP} \Phi$, Val $f=A, B=\{x:(\exists y)(x<y \&$ $f(y) \leqslant f(x))\}$. Тогда $A \cup B={ }^{*} \omega$.

ДокАЗАтЕЛЬСтво. Известно, что $\bar{B}$ - ретрассируемоемножество и $A \equiv_{T} B$. В силу леммы 1 из [4] $B$ - $Q$-полное множество, а согласно лемме Арсланова [12] всякое РП надмножество множества $B$ с бесконечным дополнением является $Q$-полным. Поэтому в силу теоремы 10 из [5] $B \nsubseteq H$ и $H \nsubseteq B$. Следовательно, $\bar{H} \subseteq * B$ (в противном случае $B \cup H$ будет $Q$-полньм гипергиперпростым, что невозможно). Но по условию $A \subset_{m} H$, поэтому $\bar{A} \subseteq{ }^{*} B$ и $A \cup B={ }^{*} \omega$. Предложение доказано.

Для каждого множества $A \subseteq \omega$ обозначим через $L(A)$ множество всех РП надмножеств множества $A$, а через $L_{r}(A)$ множество $\{A \cup R: R$ - рекурсивное множество $\}$.

Класс $\mathscr{A}$ подмножеств множества $\omega$ называется кофинальным $[1]$, если для каждого РП множества $A$ с бесконечным дополнением существует множество $B \in \mathscr{A}$ такое, что $A \subseteq B$.

Говорят, что РП множество $A$ с бесконечным дополнением имеет кофинальные гиперзиперпростые надмножества [1], если класс гипергиперпростых множеств является кофинальньм в $L(A)$.

В дальнейшем мы будем придерживаться следующих обозначений:

Max - класс максимальных множеств;

$Q$-Мах - класс квазимаксимальных множеств;

$R$-Мах - класс $r$-максимальных множеств;

$H H$ - класс гипергиперпростых множеств;

$M S_{H H}-$ класс мажорных подмножеств гипергиперпростых множеств;

$M S_{H H}^{1}-$ класс $M S_{H H} \cup H H$;

$S H$ - класс РП множеств с кофинальными гипергиперпростыми надмножества;

$H H_{\approx_{r}}-$ класс гипергиперпростых множеств по $\left(\bmod _{\approx_{r}}\right)$;

$s H S$ - класс строго гиперпростьх множеств;

$f s H S$ - класс конечно строго гиперпростых множеств;

$D S-$ класс плотно простых множеств;

$H S$ - класс гиперпростых множеств;

$S$ - класс простых множеств.

Определения всех этих классов простьх множеств можно найти в [1] и [3].

Для приведенньх выше классов простых множеств имеем следующую диаграмму (см. рис. 1) взаимного расположения. (Если $\mathscr{A}$ и $\mathscr{B}-$ классы множеств, то $\mathscr{A} \rightarrow \mathscr{B}$ обозначает, что $\mathscr{A}$ есть подкласс класса $\mathscr{B}$.)

КЛАССИФИКАЦИОННАЯ ТЕОРЕМА (см. [1], [2, теорема 2]). Имеют место следующие утверждения.

1) Все включения в приведенной выше диаграмме справедливы и являются собственными.

2) Другие включения между этими классами не выполняются.

В работе [1] дана следующая характеристика класса $H H_{\approx_{r}}$. Пусть $A-\mathrm{PП} \mathrm{множес-}$ тво с бесконечным дополнением. Тогда $A \in H H_{\approx_{r}}$ тогда и только тогда, когда

$$
(\forall X \in L(A))\left(\exists Y \in L_{r}(A)\right)\left(Y \subset_{m} X \vee Y={ }^{*} X\right)
$$




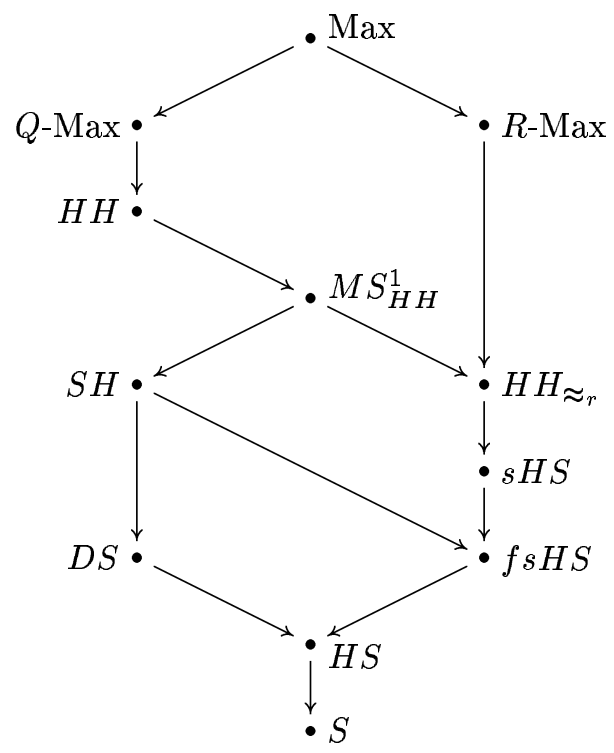

Рис. 1

Пусть $f-$ ОР $\Phi$ и $D_{x}-$ конечное множество с каноническим индексом $x$. Говорят, что $f$ определяет сильную таблииу $\left\{D_{f(x)}\right\}_{x \in \omega}$, если

$$
(\forall x)(\forall y)\left(x \neq y \Longrightarrow D_{f(x)} \cap D_{f(y)}=\varnothing\right) .
$$

Обозначим через $A \oplus B$ множество $\{2 x: x \in A\} \cup\{2 x+1: x \in B\}$. Пусть $\mathscr{K}=\left\{D S, s H S, f s H S, S H, H H_{\approx_{r}}, M S_{H H}^{1}\right\}$.

Лемма 2. Для любого $\mathfrak{A} \in \mathscr{K}$ и для любых $A, B \in S$ имеем

$$
A, B \in \mathfrak{A} \Longleftrightarrow A \oplus B \in \mathfrak{A} .
$$

ДокаЗАТЕЛЬСтво. Предварительно покажем, что если $C \in H H_{\approx_{r}}$, то $C \oplus C \in$ $H H_{\approx_{r}}$. Пусть $X \in L(C \oplus C)$. Тогда $C \subseteq\{x: 2 x \in X\}$ и $C \subseteq\{x: 2 x+1 \in X\}$. Так как $C \in H H_{\approx_{r}}$, то существуют $Y_{1}, Y_{2} \in L_{r}(C)$ такие, что $Y_{1} \subset_{m}\{x: 2 x \in X\}$ или $Y_{1}={ }^{*}\{x: 2 x \in X\}$ и $Y_{2} \subset_{m}\{x: 2 x+1 \in X\}$ или $Y_{2}={ }^{*}\{x: 2 x+1 \in X\}$.

Рассмотрим отдельно все возможные случаи.

1. $Y_{1} \subset_{m}\{x: 2 x \in X\}$ и $Y_{2} \subset_{m}\{x: 2 x+1 \in X\}$. Тогда $Y_{1} \oplus Y_{2} \subset_{m} X$. Действительно, если для некоторого РП множества $W \quad X \cup W=\omega$, то $Y_{1} \cup\{x: 2 x \in W\}=^{*} \omega$ и $Y_{2} \cup\{x: 2 x+1 \in W\}={ }^{*} \omega$. Отсюда $Y_{1} \oplus Y_{2} \cup W={ }^{*} \omega$.

2. $Y_{1}={ }^{*}\{x: 2 x \in X\}$ и $Y_{2}={ }^{*}\{x: 2 x+1 \in X\}$. Тогда $Y_{1} \oplus Y_{2}={ }^{*} X$.

3. $Y_{1} \subset_{m}\{x: 2 x \in X\}$ и $Y_{2}=^{*}\{x: 2 x+1 \in X\}$. Тогда $Y_{1} \oplus Y_{2} \subset_{m} X$. Действительно, пусть для некоторого РП множества $W \quad X \cup W=\omega$. Тогда имеем $Y_{1} \cup\{x: 2 x \in W\}={ }^{*} \omega$ и $Y_{2} \cup\{x: 2 x+1 \in W\}={ }^{*} \omega$. Отсюда $Y_{1} \oplus Y_{2} \cup W={ }^{*} \omega$, т.е. $Y_{1} \oplus Y_{2} \subset_{m} X$. Очевидно, что если $Y_{1}, Y_{2} \in L_{r}(C)$, то $Y_{1} \oplus Y_{2} \in L_{r}(C \oplus C)$.

Для остальных классов из $\mathscr{K}$ легко проверяется, что если множество $C$ принадлежит некоторому из этих классов, то $C \oplus C$ принадлежит этому же классу. Известно 
(см. [1] и [12]), что для любого $\mathfrak{A} \in \mathscr{K} \mathfrak{A}$ замкнут относительно конечных пересечений и простых надмножеств. Поэтому допустим, что $\mathfrak{A} \in \mathscr{K}$ и $A, B \in \mathfrak{A}$. Тогда $A \cap B \in \mathfrak{A}$ и $(A \cap B) \oplus(A \cap B) \in \mathfrak{A} ;(A \cap B) \oplus(A \cap B) \subseteq A \oplus B$, поэтому $A \oplus B \in \mathfrak{A}$.

Обратную импликацию будем доказьвать для каждого класса из $\mathscr{K}$ в отдельности.

1. Пусть $A, B \in S, A \oplus B \in D S$ и $A \notin D S$. Согласно результатам Робинсона [13] бесконечное множество $X$ является плотно иммунным тогда и только тогда, когда для любой сильной таблицы $\left\{D_{f(x)}\right\}_{x \in \omega}$ существует число $n \in \omega$ такое, что для любого $m \geqslant n D_{f(m)} \cap X$ содержит менее чем $m$ элементов. Поэтому для множества $A$ сушествует сильная таблица $\left\{D_{f(x)}\right\}_{x \in \omega}$ такая, что

$$
(\exists \stackrel{\infty}{m})\left(D_{f(m)} \cap \bar{A} \text { содержит по крайней мере } m\right. \text { элементов), }
$$

где символом $\exists \stackrel{\infty}{m}$ обозначается выражение "существует бесконечно много $m$ ".

Рассмотрим сильную таблицу $\left\{D_{g(m)}\right\}_{m \in \omega}$, где

$$
(\forall m)\left(D_{g(m)}=\left\{2 x: x \in D_{f(m)}\right\}\right) .
$$

Тогда

$$
(\exists \stackrel{\infty}{m})\left(D_{g(m)} \cap \overline{A \oplus B} \text { содержит по крайней мере } m\right. \text { элементов). }
$$

Следовательно, $A \oplus B \notin D S$. Полученное противоречие доказьвает, что $A \in D S$.

2. Пусть $A, B \in S, A \oplus B \in s H S$ и $A \notin s H S$. Тогда сушествует ОР $\Phi f$ такая, что

1) $(\forall x)\left(W_{f(x)} \cap \bar{A} \neq \varnothing\right)$;

2) $(\forall x)(\forall y)\left(x \neq y \Longrightarrow W_{f(x)} \cap W_{f(y)}=\varnothing\right)$;

3) $\bigcup_{x \in \omega} W_{f(x)}=\omega$.

Определим ОРФ $g$ равенством

$$
W_{g(x)}=\left\{2 y: y \in W_{f(x)}\right\} \cup\left\{2 y+1: y \in W_{f(x)}\right\}
$$

Тогда существование функции $g$ доказывает, что $A \oplus B \notin s H S$. Противоречие. Поэтому $A \in s H S$.

Точно так же доказывается, что если $A, B \in S$ и $A \oplus B \in f s H S$, то $A, B \in f s H S$.

3. Пусть $A, B \in S, A \oplus B \in S H$ и $A \notin S H$. Допустим, что $C-$ РП множество, $A \subseteq C, \bar{C}$ бесконечно и $C$ не содержится в гипергиперпростом множестве. Тогда $A \oplus B \subseteq C \oplus B$ и $C \oplus B \subseteq H$, где $H$ - гипергиперпростое множество. Отсюда имеем $A \subseteq C \subseteq C_{1}=\{x: 2 x \in H\}$. Очевидно, что $C_{1}$ не является гипергиперпростым множеством. Поэтому существует ОР $\Phi f$ такая, что

$$
\begin{gathered}
(\forall x)\left(W_{f(x)} \cap \bar{C}_{1} \neq \varnothing\right), \\
(\forall x)(\forall y)\left(x \neq y \Longrightarrow W_{f(x)} \cap W_{f(y)}=\varnothing\right) .
\end{gathered}
$$

Определим ОРФ $g$ равенством

$$
W_{g(x)}=\left\{2 y: y \in W_{f(x)}\right\} \text {. }
$$


Тогда существование функции $g$ противоречит гипергиперпростоте множества $H$. Полученное противоречие доказывает, что если $A \oplus B \in S H$, то $A, B \in S H$.

4. Пусть $A, B \in S, A \oplus B \in H H_{\approx_{r}}$ и $A \notin H H_{\approx_{r}}$. Тогда

$\left(\exists X \in L_{r}(A)\right)\left(\forall Y \in L_{r}(A)\right)\left(Y \not \subset_{m} X \quad \& Y \neq^{*} X\right) \quad$ и $\quad A \oplus B \subseteq X \oplus B$.

Поэтому

$$
\left(\exists Y_{1} \in L_{r}(A \oplus B)\right)\left(Y_{1} \subset_{m} X \oplus B \vee Y_{1}={ }^{*} X \oplus B\right) .
$$

Рассмотрим отдельно эти случаи.

Пусть $Y_{1}=A \oplus B \cup R$, где $R$ - рекурсивное множество. Если $Y_{1}={ }^{*} X \oplus B$, то $\left\{x: 2 x \in Y_{1}\right\}={ }^{*} X$. Обозначим через $R_{1}$ множество $\{x: 2 x \in R\}$. Тогда $A \cup R_{1}={ }^{*} X$, что противоречит соотношению $(*)$.

Пусть

$$
Y_{1}=A \oplus B \cup R \subset_{m} X \oplus B, \quad Y_{2}=\{x: 2 x \in A \oplus B \cup R\}=A \cup R_{1} .
$$

Если $Y_{2}=A \cup R_{1}{ }^{*} X$, то опять получается противоречие. Допустим, что $\left|X \backslash\left(A \cup R_{1}\right)\right|$ $=\infty$, и покажем, что $A \cup R_{1} \subset_{m} X$.

Пусть $X \cup W=\omega$, где $W-$ РП множество. Тогда

$$
X \oplus B \cup W \oplus W \cup\{2 x+1: x \in X\}=\omega .
$$

Отсюда и из соотношения $(* *)$ получаем, что

$$
A \oplus B \cup R \cup W \oplus W \cup\{2 x+1: x \in X\}={ }^{*} \omega .
$$

Но из этого следует, что $A \cup R_{1} \cup W={ }^{*} \omega$. Итак, получили, что если $A \oplus B \in H H_{\approx_{r}}$, то $A, B \in H H_{\approx_{r}}$.

5. Пусть $A, B \in S$ и $A \oplus B \in M S_{H}^{1}$. Если $A \oplus B \in H H$, то в силу леммы 3 из [15] $A, B \in H H$. Допустим, что $A \oplus B \subset_{m} B_{1}$, где $B_{1}$ - гипергиперпростое множество; $A \subset A_{1}^{\prime}=\left\{x: 2 x \in B_{1}\right\}$ и $B \subset B_{1}^{\prime}=\{x: 2 x+1 \in B\}$. Очевидно, что $A_{1}^{\prime}, B_{1}^{\prime} \in H H$. Если $A={ }^{*} A_{1}^{\prime}$, то $A \in M S_{H}^{1}$. Пусть $\left|A_{1}^{\prime} \backslash A\right|=\infty$. Покажем, что $A \subset_{m} A_{1}^{\prime}$. Допустим, что для некоторого РП множества $W A_{1}^{\prime} \cup W=\omega$. Тогда $A_{1}^{\prime} \oplus B_{1}^{\prime} \cup W \oplus W \cup\{2 x+1: x \in A\}=\omega$. Отсюда $A \oplus B \cup W \oplus W \cup\{2 x+1: x \in A\}=\omega$. Тогда $A \cup W={ }^{*} \omega$, т.е. $A \in M S_{H}^{1}{ }_{H}$. Аналогично проверяется, что $B \in M S_{H}^{1}$. Лемма доказана.

Teоpema 2. 1) ( $\exists A)(A-Q$-полное \& $A \in S \backslash H S)$.

2) $(\exists A)(A-Q$-полное \& $A \in H S \backslash(D S \cup f s H S))$.

3) $(\exists A)(A-Q$-полное \& $A \in D S \backslash f s H S)$.

4) $(\exists A)(A-Q$-полное \& $A \in f s H S \backslash(S H \cup s H S))$.

5) $(\exists A)(A-Q$-полное \& $A \in S H \backslash s H S)$.

6) $(\exists A)\left(A-Q\right.$-полное \& $\left.A \in s H S \backslash H H_{\approx_{r}}\right)$.

7) $(\exists A)\left(A-Q\right.$-полное \& $A \in H H_{\approx_{r}} \backslash\left(M S_{H}^{1} \cup R\right.$-Max $\left.)\right)$.

8) $(\exists A)(A-Q$-полное \& $A \in R$-Max).

9) $(\exists A)\left(A-Q\right.$-полное \& $\left.A \in M S_{H}^{1}\right)$. 
ДокАЗАТЕльство. 1) Известно [3, с. 207], что гиперпростое множество не может быть $W$-полным и существует $C$-полное простое множество $A$. Поэтому $A$ является искомьм множеством.

2) Пусть $B-Q$-полное гиперпростое множество с ретрассируемым дополнение (такое существует). Тогда $B \notin f s H S$, так как известно [16], что конечно строго гиперпростое множество не имеет ретрассируемого дополнения. Рассмотрим множество $B \oplus C$, где $C \in H S \backslash D S$. Тогда $B \oplus C$ является $Q$-полным множеством и в силу леммы 2 $B \oplus C \in H S \backslash(D S \cup f s H S)$.

3) Известно, что если $B-W$-полное РП множество, $f$ - одно-однозначная ОР $\Phi$, Val $f=B$, то множество $C=\{x:(\exists y)(y>x \quad \& \quad f(y) \leqslant f(x))\}$ является плотно простьм множеством с ретрассируемьм дополнением; $B \equiv_{T} C$. Поэтому по лемме 1 из [4] множество $C$-полно. С другой стороны, конечно строго гиперпростое множество не имеет ретрассируемого дополнения. Следовательно, $C \notin f s H S$.

4) Пусть $B \in f s H S \backslash S H$. Без ограничения общности допустим, что $B$ не содержится в гипергиперпростом множестве. Тогда в силу теоремы 10 из [5] существует $Q$-полное множество $B_{1}$ такое, что $B \subseteq B_{1}$ и $B_{1}$ не содержится в гипергиперпростом множестве. Поэтому $B_{1} \notin S H$. Пусть $B_{2} \in f s H S \backslash s H S$. Тогда $B_{1} \oplus B_{2}$ является $Q$-полным и в силу леммы $2 B_{1} \oplus B_{2} \in f s H S \backslash(S H \cup s H S)$.

5) Пусть $A_{1} \in S H \backslash s H S, B-Q$-полное мажорноеподмножества множества $A_{1}$. Тогда $B \in S H \backslash s H S$. Если $B \subset C,\left|A_{1} \backslash C\right|=\infty$ и $C \cup A_{1}={ }^{*} \omega$, то $B \cup C={ }^{*} \omega$. Поэтому невозможно, чтобы $\left|A_{1} \backslash C\right|=\infty$ и $C \cup A_{1}={ }^{*} \omega$.

6) Пусть $A_{1} \in s H S \backslash H H_{\approx_{r}}$ и $A_{1} \subseteq B$, где $B-Q$-полное РП множество (такое существует по теореме 10 из [5] или по теореме 5 из [6]). Тогда $A_{1} \oplus B$ является $Q$-полным и в силу леммы $2 A_{1} \oplus B \in s H S \backslash H H_{\approx_{r}}$.

7) Пусть $A_{1} \in H H_{\approx_{r}} \backslash M S_{H H}^{1}, B \in H H_{\approx_{r}} \backslash R$-Мах. Тогда

$$
A_{1} \oplus B \in H H_{\approx_{r}} \backslash\left(M S_{H H}^{1} \cup R-\operatorname{Max}\right)
$$

Действительно, в силу леммы $2 A_{1} \oplus B \in H H_{\approx_{r}} \backslash M S_{H H}^{1}$ и нетрудно проверить, что если $A_{1} \oplus B \in R$ - Max, то $A_{1}, B \in R$ - Max.

Пусть $C=A_{1} \oplus B, E-Q$-полное РП множество и $C \subseteq E$ (такое существует по теореме 10 из [5] или по теореме 5 из [6]). Тогда $C \oplus E-Q$-полное множество и по лемме $2 C \oplus E \in H H_{\approx_{r}}$ и $C \oplus E \notin M S_{H}^{1}$. Если $C \oplus E \in R$-Max, то $C, E \in R$-Max. Поэтому $C \oplus E \notin R$ - Max.

8) и 9) непосредственно вытекают из теоремы 1 и из того, что мажорное подмножество $r$-максимального множества является $r$-максимальньм. Теорема доказана.

\section{СПИСОК ЦИТИРОВАННОЙ ЛИТЕРАТУРЫ}

[1] Herrmann E. Classes of simple sets, filter properties, and their mutual position // Seminarber. Humbolt-Univ. Berlin. Sekt. Math. 1984. №60. P. 60-72.

[2] Оманадзе Р.Ш. Классы рекурсивно перечислимых множеств и $Q$-сводимость // Матем. заметки. 1989. Т. 45. №2. С. 79-82.

[3] Роджерс Х. Теория рекурсивных функций и эффективная вычислимость. М.: Мир, 1972.

[4] Марченков С. С. Об одном классе неполных множеств // Матем. заметки. 1976. Т. 20. № 4. C. 473-478. 
[5] Соловьев В. Д. Q-сводимость и гипергиперпростые множества // Вероятностные методы и кибернетика. Вып. 10-11. Казань: КазГУ, 1974. С. 121-128.

[6] Gill J.T., Morris P.H. On subcreative sets and $S$-reducibility // J. Symb. Logic. 1974. V. 39. № 4. P. 669-677.

[7] Lachlan A. H. On the lattice of recursively enumerable sets // Trans. Amer. Math. Soc. 1968. V. 130. № 1. P. 1-37.

[8] Herrmann E. Die Verbandseigenschaften der rekursiv aufzählbaren Mengen // Seminarber. Humbolt-Univ. Berlin. Sekt. Math. 1981. № 36.

[9] Blum M., Marques I. On complexity properties of recursively enumerable sets // J. Symb. Logic. 1973. V. 38. № 4. P. 579-593.

[10] Lerman M. Some theorems of $r$-maximal sets and major subsets of recursively enumerable sets // J. Symb. Logic. 1971. V. 36. P. 193-215.

[11] Дёгтев А.Н. Об m-степенях простых множеств // Алгебра и логика. 1972. Т. 11. № 2. С. $130-139$.

[12] Арсланов М. М. Об одном классе гиперпростых неполных множеств // Матем. заметки. 1985. Т. 38. №6. С. $872-874$.

[13] Дёгтев А.Н. Некоторые классы гипериммунных множеств // Алгебраические системы. Иркутск, 1976. С. 21-36.

[14] Robinson R. W. Simplicity of recursively enumerable sets // J. Symb. Logic. 1967. V. 32. № 2. P. 162-172.

[15] Морозов А. С. Об одном классе рекурсивно перечислимых множеств // Сиб. матем. ж. 1987. Т. 23. № 2. С. $124-128$.

[16] Дёгтев А.Н. Наследственные множества и табличная сводимость // Алгебра и логика. 1972. Т. 11. № 3. С. 257-269.

Тбилисский государственный университет им. И. А. Джавахишвили

Поступило

Институт прикладной математики им. И. Н. Векуа

E-mail : omanr@viam.hepi.edu.ge

31.03 .1998

Исправленный вариант

01.02.2001 